\title{
In situ Observation of 15 mol\% Ba-Substituted Strontium Tantalate up to 1773 K by Ultraviolet Laser Raman Spectroscopy
}

\author{
Hirotaka Fujimori $^{1 *}$, Ippei Kawanishi ${ }^{1}$ and Nobuhiro Matsushita ${ }^{2}$ \\ 1 Department of Applied Chemistry, Yamaguchi University, 2-16-1 Tokiwadai, Ube, Yamaguchi 755-8611 Japan \\ 2 Materials and Structures Laboratory, Tokyo Institute of Technology, 4259 Nagatsuta, Midori-ku, Yokohama, \\ Kanagawa 226-8503 Japan \\ * Corresponding author: Fax: 81-836-85-9672, e-mail: hiro@hiro-fuji.net, fujimori@yamaguchi-u.ac.jp
}

\begin{abstract}
Raman spectra of 15 mol\% Ba-substituted $\mathrm{Sr}_{2} \mathrm{Ta}_{2} \mathrm{O}_{7}$ have been successfully obtained at temperatures up to $1773 \mathrm{~K}$ by a continuous-wave ultraviolet Raman spectroscopic system. The Raman spectra do not change significantly with an increase in temperature, despite the $P 2_{1} \rightarrow$ $\mathrm{Cmcm}$ transition. The present result is described in terms of the parameter related to temperature variation at constant pressure, defined in a similar way to the Gruneisen parameter. Raman bands located above $220 \mathrm{~cm}^{-1}$ show smaller values of the parameter than lower frequency modes, suggesting that these higher frequency modes are assigned to $\mathrm{TaO}_{6}$ internal modes. Due to the strong $\mathrm{Ta}-\mathrm{O}$ chemical bonds, the dipole moments in the $\mathrm{TaO}_{6}$ octahedra and the ferroelectric polarization in the whole crystal lattice can be induced by the Ba substitution without large deviation of the $\mathrm{Ta}-\mathrm{O}-\mathrm{Ta}$ bond angles from $180^{\circ}$, which enhances the photocatalytic performance.
\end{abstract}

Key words: perovskite, tantalate, Raman scattering, high temperature

\section{INTRODUCTION}

Photocatalytic water splitting has been studied from a view point of light energy conversion. NiO-supported strontium tantalate, $\mathrm{Sr}_{2} \mathrm{Ta}_{2} \mathrm{O}_{7}$, is an attractive substance, because it has been known as a photocatalytic material to decompose $\mathrm{H}_{2} \mathrm{O}$ into $\mathrm{H}_{2}$ [1,2]. Preparation of solid solution is one of the ways to enhance photocatalytic activity. Thus, we synthesized $\mathrm{Ba}$-substituted $\mathrm{Sr}_{2} \mathrm{Ta}_{2} \mathrm{O}_{7}$ and confirmed a solubility limit of $\mathrm{Ba}$ is $20 \mathrm{~mol} \%$ for $\mathrm{Sr}$. The enhancement of the photocatalytic activity was observed to be three times as high as that of unsubstituted $\mathrm{Sr}_{2} \mathrm{Ta}_{2} \mathrm{O}_{7}$ when $\mathrm{Ba}$ was substituted for 20 $\mathrm{mol} \%$ of $\mathrm{Sr}$ [3].

$\mathrm{Sr}_{2} \mathrm{Ta}_{2} \mathrm{O}_{7}$ has a layered perovskite structure, whose formula can be converted into $\mathrm{Sr}_{(1-1 / 7)} \mathrm{Ta}_{(1-1 / 7)} \mathrm{O}_{3}$. It denotes a layered defective perovskite $A B \mathrm{O}_{3}$ form with the " $A$ " and " $B$ " cation defects every four perovskite units as shown in Fig. 1. The space group of $\mathrm{Sr}_{2} \mathrm{Ta}_{2} \mathrm{O}_{7}$ is monoclinic $P 2_{1} / m[4,5]$ or orthorhombic $\mathrm{Cmcm}$ [6] at room temperature. When the space group is $P 2_{1} / m$, it is reported to transform into an ideal layered perovskite structure with $\mathrm{Cmcm}$ around $443 \mathrm{~K}$ by electron microscopy and diffraction [4,5], Raman spectroscopy [7] and dielectric dispersion [8]. The nature of chemical bonds in a substance is one of the most important keys to decisions on the physical and chemical properties. Introducing structural perturbations into the structure of a compound gives valuable insights into the property of the chemical bonds. Variations in pressure and temperature are available means to introduce structural perturbations. X-ray diffraction (XRD) reveals structural variation through static atomic displacements in an equilibrium state. On the other hand, Raman scattering approaches the issue of a structural perturbation from a dynamic point of view, through the frequency shifts of vibrational modes. The vibrational spectra recorded at high temperature and high pressure reveal detailed information on the bonds and the physical properties, and also provide constraints on thermodynamic properties in a phase equilibrium at temperature and pressure beyond the range of direct thermodynamic measurement. However, the measurable region of temperature is limited under conventional Raman spectroscopy using visible-region excitation $[9,10]$.

We have already obtained the successful Raman spectra of $\mathrm{Ca}_{2} \mathrm{SiO}_{4}$ at high temperatures up to $1723 \mathrm{~K}$ [11], using a continuous-wave (CW) ultraviolet (UV) Raman spectroscopic system designed to measure the Raman scattering from substances at high temperature [9]. Moreover, we have assigned each Raman band by calculating the Gruneisen parameter, $\gamma_{i T}$, related to pressure variation at constant temperature, and the

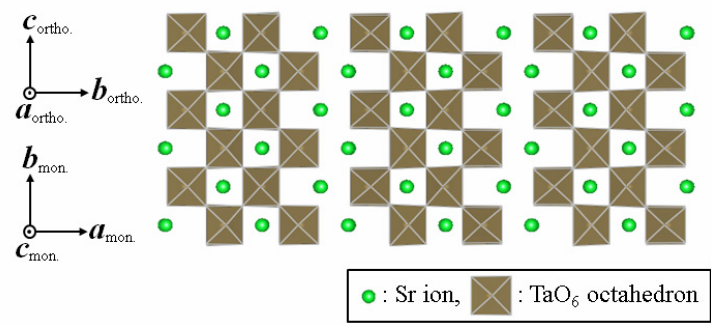

Fig. 1 Schematic drawing of the crystal structure of $\mathrm{Sr}_{2} \mathrm{Ta}_{2} \mathrm{O}_{7}$. The space group of $\mathrm{Sr}_{2} \mathrm{Ta}_{2} \mathrm{O}_{7}$ is monoclinic $P 2_{1} / m \quad[4,5]$ or orthorhombic $\mathrm{Cmcm}$ [6] at room temperature. The axes of both monoclinic $P 2_{1} / m$ and orthorhombic $\mathrm{Cmcm}$ are indicated in the figure. 
corresponding parameter, $\gamma_{i P}$, related to temperature variation at constant pressure. The intrinsic anharmonic parameter was derived from these two parameters [11] Anharmonic effect can substantially affect the physical and chemical properties of materials.

In the present study, we observed Raman spectra of $15 \mathrm{~mol} \%$ Ba-substituted strontium tantalate at high temperatures up to $1773 \mathrm{~K}$, which have not yet been reported. We selected the solid solution with $15 \mathrm{~mol} \%$ of $\mathrm{Ba}$ substitution to avoid the possibility of existence of other phases than $\mathrm{Sr}_{2} \mathrm{Ta}_{2} \mathrm{O}_{7}$ in the vicinity of the solubility limit at $20 \mathrm{~mol} \%$ of $\mathrm{Ba}$. The result is discussed here from a point of view of properties of chemical bonds in terms of the parameter $\gamma_{i P}$, defined in a similar way to the Gruneisen parameter. Moreover, the assignments of the respective bands in Raman spectra of $15 \mathrm{~mol} \%$ Ba-substituted $\mathrm{Sr}_{2} \mathrm{Ta}_{2} \mathrm{O}_{7}$ are predicted experimentally through the properties of the chemical bonds. This study will make a step toward an investigation into the mechanism on the enhancement of photocatalytic performance in $\mathrm{Sr}_{2} \mathrm{Ta}_{2} \mathrm{O}_{7}$ as well as our study on $\mathrm{Sr}_{2} \mathrm{Nb}_{2} \mathrm{O}_{7}$ [12].

\section{EXPERIMENTS}

2.1 Synthesis of unsubstituted and $15 \mathrm{~mol} \%$ Ba-substituted strontium tantalate powders

Compositionally homogeneous samples are important for measurement of Raman spectra. Powders of unsubstituted and $15 \mathrm{~mol} \%$ Ba-substituted strontium tantalates, $\left(\mathrm{Sr}_{1-x} \mathrm{Ba}_{x}\right)_{2} \mathrm{Ta}_{2} \mathrm{O}_{7}: \quad x=0$ and 0.15 , were synthesized using the polymerizable complex method, which is well suited to the synthesis of compositionally homogeneous strontium tantalates due to different metal ions dispersing homogeneously at an atomic level $[2,3,13] . \mathrm{TaCl}_{5}$ (Junsei Chemical Co., Ltd., Japan) was used as a source of tantalum, which was precisely weighed and dissolved into methanol at room temperature in $\mathrm{N}_{2}$ gas in order to avoid hydrolysis. The $\mathrm{TaCl}_{5}$ methanol solution was added into a mixture of ethylene glycol (Kanto Chemical Co., Ltd., Japan) and anhydrous citric acid (Wako Pure Chemical Industries, Ltd., Japan). $\mathrm{SrCO}_{3}(69.86 \%$ as $\mathrm{SrO}$ content, Seimi Chemical Co., Ltd., Japan) and $\mathrm{BaCO}_{3}(78.48 \%$ as $\mathrm{BaO}$, Seimi Chemical Co., Ltd., Japan) were added in a molar ratio of $\mathrm{Sr}: \mathrm{Ba}: \mathrm{Ta}=2(1-x): 2 x: 2$ in consideration of a metal ion content in each chemical reagent. Three times the amount of anhydrous citric acid was used to $\mathrm{Ta}, \mathrm{Sr}$ and $\mathrm{Ba}$ when the charge balance was taken into account, assuming charged valences of $\mathrm{Ta}, \mathrm{Sr}, \mathrm{Ba}$ and $\mathrm{C}_{6} \mathrm{H}_{8} \mathrm{O}_{7}$ to be $+5,+2,+2$ and -3 , respectively. Above procedures were carried out with stirring at $\sim 353 \mathrm{~K}$. After adding all needed chemicals, the mixture was kept at $\sim 353 \mathrm{~K}$ with stirring until all chemicals added were dissolved into the solution to become transparent. The resulting clear colorless solution was gradually heated up to $\sim 423 \mathrm{~K}$ while stirring to promote polymerization. On continuously heating at $\sim 423 \mathrm{~K}$, the solution became highly viscous with a change in color from colorless to yellow and eventually gelled into a transparent brown, glassy resin. Noteworthy is that no visible formation of precipitation or turbidity was observed during the polymerization and gelation. The charring resin was carried out in a mantle heater at $\sim 723 \mathrm{~K}$ to remove most of organics. The resulting solid white mass was ground into a powder with an agate mortar. The powder was calcined at $1073 \mathrm{~K}$ for $2 \mathrm{~h}$ in air on an alumina boat followed by natural cooling to room temperature in a furnace. The powder was ground and then calcined at $1273 \mathrm{~K}$ for $24 \mathrm{~h}$ in the same way.

2.2 Second harmonic generation (SHG)

The powder second harmonic generation (SHG) technique provides a sensitive test for non-centrosymmetry. A signal of each sample was measured by the powder technique of Kurtz and Perry [14]. A powder sample was densely packed between two transparent glass slides. The SHG signal was generated by irradiating the powder sample by a femtosecond-pulsed-laser beam. The source beam consists of a mode-locked Ti: Sapphire laser (wavelength $\sim 800 \mathrm{~nm}$, repetition rate $\sim 82 \mathrm{MHz}$, pulse width $\sim 80$ fs, Tsunami, Spectra-Physics, Newport Corporation, USA) pumped by a CW YAG laser (wavelength $532 \mathrm{~nm}$, Millennia, Spectra-Physics, Newport Corporation, USA). The beam was focused by a quartz lens (L1, $f=100 \mathrm{~mm}$ ) to $\sim 0.1 \mathrm{~mm}$ diameter spot on the surface of the powder sample. The output light, containing the scattered fundamental $(\sim 800 \mathrm{~nm})$ and the SHG signal $(\sim 400 \mathrm{~nm})$ wavelengths, was gathered by a quartz lens (L2, $f=100 \mathrm{~mm}$ ) placed diagonally in front of the sample. A violet filter (transmission from near-ultraviolet to blue light, V-44, Toshiba glass Co. Ltd., Japan) was used in order to reduce the strong scattered fundamental wavelength in the output light. The SHG signal was collected by an optical fiber probe (Hamamatsu Photonics K. K., Japan) and was dispersed with a spectrometer (Imaging Spectrograph 250is, Chromex Inc., USA), and then detected with a charge-coupled device (CCD) (M6296-01, Hamamatsu Photonics K. K., Japan).

2.3 In situ observation by ultraviolet laser Raman spectroscopy

Raman measurements were performed for unsubstituted strontium tantalate at room temperature and for $15 \mathrm{~mol} \%$ Ba-substituted one at various temperatures using a continuous-wave UV Raman spectroscopic system, which is based on an ultraviolet argon-ion laser $(363.8 \mathrm{~nm})$, a spatial filter, a triple monochromator and a two-dimensional CCD detector to be designed for measurement of Raman scattering from substances at high temperature [9]. We have already used UV Raman spectroscopy to observe the phase transition in $\mathrm{SrZrO}_{3}$ at $\sim 1473 \mathrm{~K}$ [15], $\mathrm{OH}$ vacancies arising in hydroxyapatite at high temperatures [16], the phase transition of hafnia at $2085 \mathrm{~K}$ [17] and an anharmonic lattice mode of $\mathrm{Ca}_{2} \mathrm{SiO}_{4}$ [11]. The $15 \mathrm{~mol} \%$ Ba-substituted strontium tantalate sample was mounted in a furnace with a Pt-Rh heater, where the sample with a size of a grain of rice was placed and then fixed onto a Pt-13\% Rh thermocouple with alumina cement. The sample was heated at a rate of $10 \mathrm{~K} / \mathrm{min}$, after which the temperature was held constant during each measurement (within $\pm 1 \mathrm{~K}$ ). Measurements up to $1773 \mathrm{~K}$ were conducted in air after a constant temperature had been maintained for $10 \mathrm{~min}$. The laser power at the tube level was set at $90 \mathrm{~mW}$. The laser spot size was about $100 \mu \mathrm{m}$ in diameter at the sample point. In view of the spectral resolution and the high spectral intensity at high temperatures, the entrance slit width was set at $100 \mu \mathrm{m}$, 
which corresponds to a full-width-at-half-maximum of $4.7 \mathrm{~cm}^{-1}$. In the region from 1573 to $1773 \mathrm{~K}$, the backgrounds of thermal emission from the sample and the furnace were measured without the laser line, and then they were subtracted from the Raman spectra measured at the high temperatures. An $\mathrm{Hg}$ line was used for Raman-shift calibration for each measurement. No artificial smoothing was used on the acquired Raman spectra.

\section{RESULTS AND DISCUSSION}

At room temperature, the powder XRD patterns of the present samples, $x=0$ and 0.15 , showed that they resemble simulated patterns based on crystal structures obtained from single crystal XRD analyses of

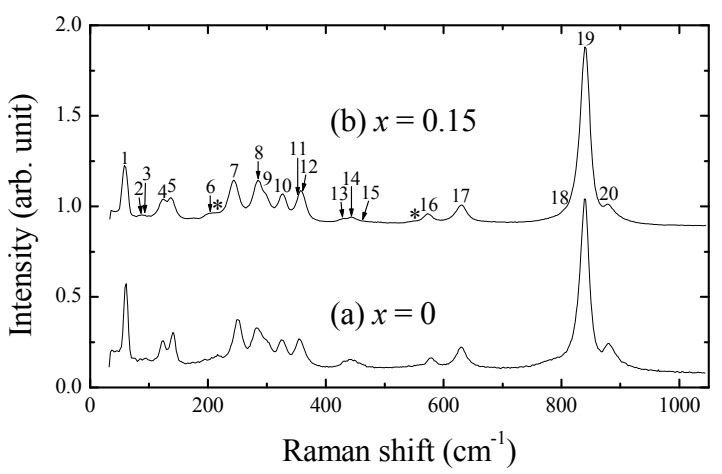

Fig. 2 Comparison of Raman spectra of $\left(\mathrm{Sr}_{1-x} \mathrm{Ba}_{x}\right)_{2} \mathrm{Ta}_{2} \mathrm{O}_{7}$ at $298 \mathrm{~K}$ between (a) $x=0$ and (b) $x=0.15$. Intensity of each spectrum is normalized by the highest intensity of each spectrum. Both spectra showed considered twenty Raman bands, $i=1$ to 20 , in order of increasing frequency, and two additional bands $(*)$. Temperature dependences of additional bands $(*)$ were impossible to be estimated due to no data points at high temperatures because they have low peak heights even at room temperature.

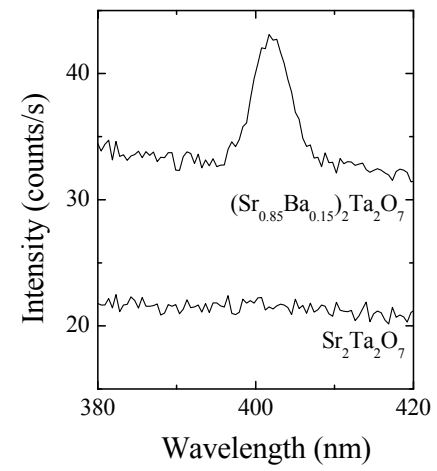

Fig. 3 Second harmonic (SH) emission of $\left(\mathrm{Sr}_{1-0.15} \mathrm{Ba}_{0.15}\right)_{2} \mathrm{Ta}_{2} \mathrm{O}_{7}$ by irradiating the powder sample by a $\sim 800 \mathrm{~nm}$ pulsed laser beam. For comparison, a measurement result of $\mathrm{Sr}_{2} \mathrm{Ta}_{2} \mathrm{O}_{7}$ is also shown in the same measurement condition, which provides no signal due to its centrosymmetric crystal structure.
$A_{2} B_{2} \mathrm{O}_{7}$-type compounds with the $P 2_{1}$ symmetry such as $\mathrm{La}_{2} \mathrm{Ti}_{2} \mathrm{O}_{7}$ [18] and $\mathrm{Ca}_{2} \mathrm{Nb}_{2} \mathrm{O}_{7}$ [19], while typical Raman spectra of $\mathrm{Sr}_{2} \mathrm{Ta}_{2} \mathrm{O}_{7}$ were recorded (Fig. 2) [20]. In other words, the Raman spectra of unsubstituted and $15 \mathrm{~mol} \%$ Ba-substituted strontium tantalates were almost the same. By means of the powder diffraction technique, centrosymmetric and non-centrosymmetric structures cannot be distinguished; the $\mathrm{Sr}_{2} \mathrm{Ta}_{2} \mathrm{O}_{7}$ with centrosymmetric $P 2_{1} / m$ shows a similar diffraction pattern to non-centrosymmetric $P 2_{1}$ because the extinction rule is the same between $P 2_{1} / m$ and $P 2_{1}$, moreover, it is impossible to confirm the breakdown of Friedel's law in the absence of a center of symmetry. The SHG results of $\left(\mathrm{Sr}_{1-x} \mathrm{Ba}_{x}\right)_{2} \mathrm{Ta}_{2} \mathrm{O}_{7}$ powders at $x=0$ and 0.15 (Fig. 3) clearly show whether there exists symmetry center in the respective crystal lattices. There exists no SHG signal in the sample of $x=0$, demonstrating centrosymmetry and suggesting a space group of $P 2_{1} / \mathrm{m}$. On the other hand, the SHG signal was clearly observed in the sample of $x=0.15$, indicating non-centrosymmetry due to displacement of cations and rotation of oxygen octahedra from an ideal layered perovskite structure with $\mathrm{Cmcm}$. Thus, the space group of the sample at $x=0.15$ is suggested to be $P 2_{1}$ like the structures of

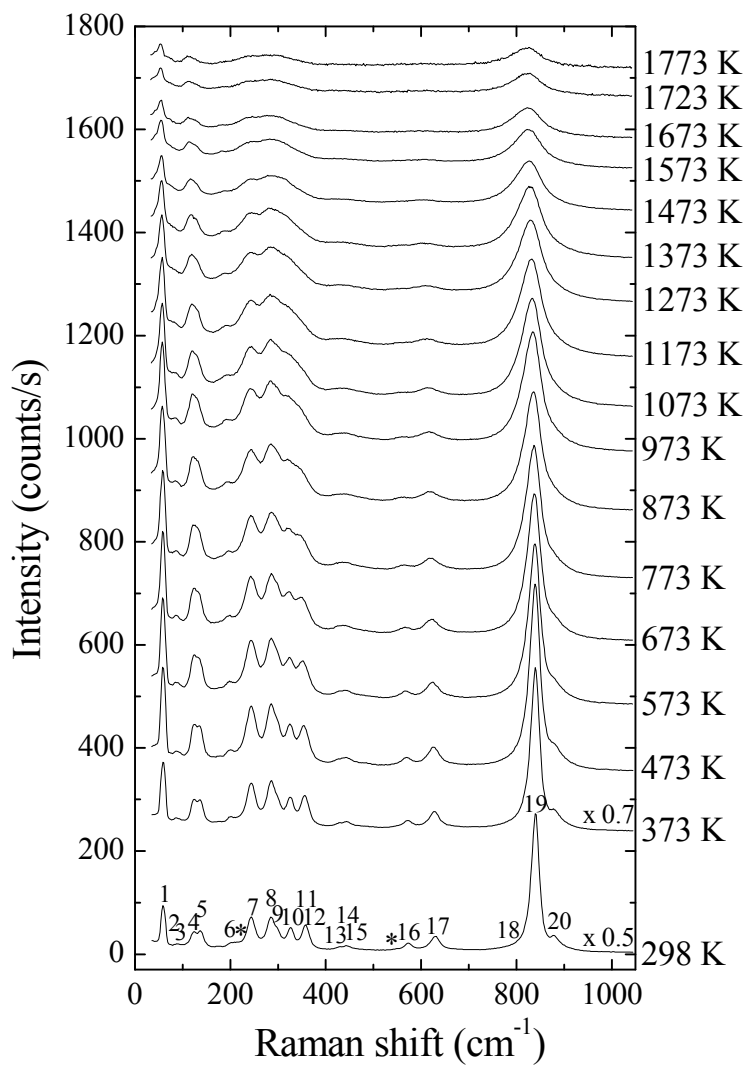

Fig. 4 Temperature dependence of the Raman spectra of $\left(\mathrm{Sr}_{0.85} \mathrm{Ba}_{0.15}\right)_{2} \mathrm{Ta}_{2} \mathrm{O}_{7}$ exited by the $363.8 \mathrm{~nm}$ UV laser line. Note scale factors applied to spectra at 298 and $373 \mathrm{~K}$. The spectra showed considered twenty Raman bands, labeled by $i=1$ to 20 in order of increasing frequency, and two additional peaks $(*)$. 
unsubstituted $\mathrm{Sr}_{2} \mathrm{Ta}_{2} \mathrm{O}_{7}$ below $166 \mathrm{~K}$ [4], $\mathrm{La}_{2} \mathrm{Ti}_{2} \mathrm{O}_{7}[18]$ and $\mathrm{Ca}_{2} \mathrm{Nb}_{2} \mathrm{O}_{7}$ [19] at room temperature. These suggestions are supported by the synchrotron XRD and neutron diffraction data of the same samples [21].

With an increase in the temperature of $\left(\mathrm{Sr}_{1-x} \mathrm{Ba}_{x}\right)_{2} \mathrm{Ta}_{2} \mathrm{O}_{7}$ at $x=0.15$, all bands broadened (Fig. 4). The spectra showed twenty Raman bands and more; $i=$ 1 to 20 , in order of increasing frequency, and two additional bands denoted by a symbol of $(*)$. The additional peaks mean the peaks whose temperature dependences were impossible to be estimated due to no data points at high temperatures because they have low peak heights even at room temperature. The twenty two Raman bands are assigned later in this paper. When taking account of a transformation on unsubstituted $\mathrm{Sr}_{2} \mathrm{Ta}_{2} \mathrm{O}_{7}$ from $P 2_{1} / m$ to $C m c m$ around $443 \mathrm{~K}[4,5]$, the $P 2_{1} \rightarrow \mathrm{Cmcm}$ phase transition of $15 \mathrm{~mol} \%$

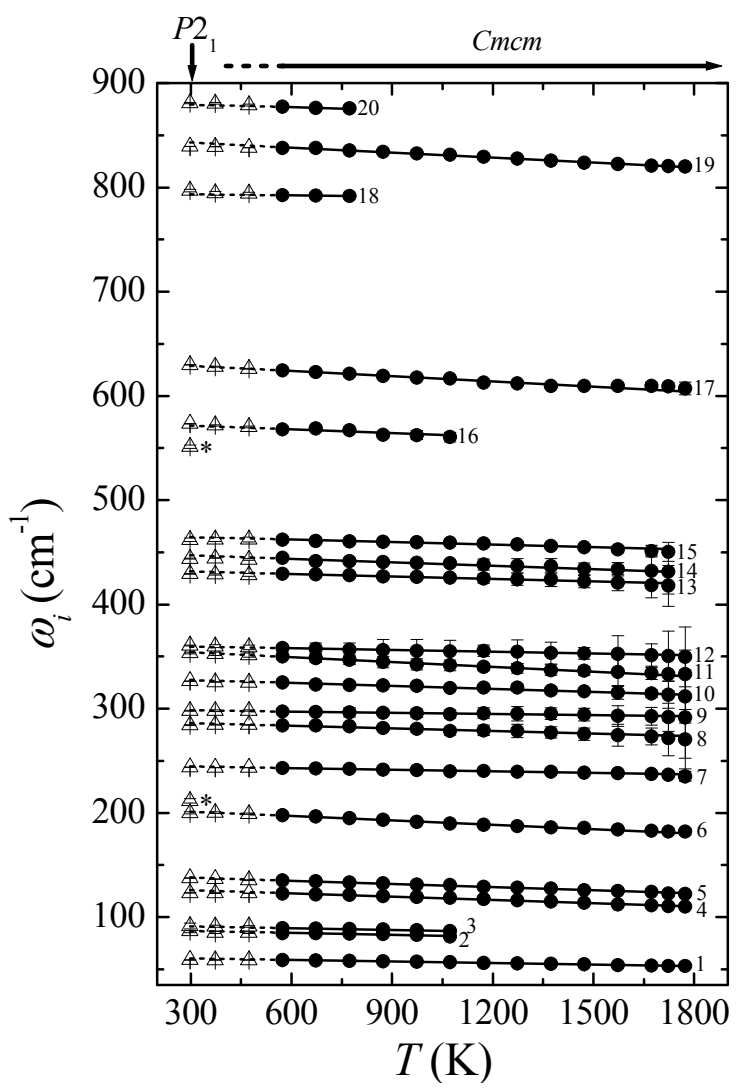

Fig. 5 Temperature dependence of the Raman bands of $\left(\mathrm{Sr}_{0.85} \mathrm{Ba}_{0.15}\right)_{2} \mathrm{Ta}_{2} \mathrm{O}_{7}$ at room pressure. Each straight solid line represents $a$ fit by least-squares regression with the weight $w=$ (error) $)^{-2}$ on $\omega_{i}$ of a considered Raman band $(i=$ 1 to 20) denoted by symbols (•) in the temperature range over $573 \mathrm{~K}$. Because good linearity of each Raman band frequency with temperature was not obtained from room temperature to $473 \mathrm{~K}$, the frequency data $(\Delta)$ were not used to fit. Each dotted line denotes extrapolation of each related solid line to $T_{0}=298 \mathrm{~K}$. Temperature dependences of some bands $(*)$ were impossible to be estimated due to no data points at high temperatures.
Ba-substituted $\mathrm{Sr}_{2} \mathrm{Ta}_{2} \mathrm{O}_{7}$ seemingly occurs at a higher temperature than room temperature, although which is not certain whether to occur directly or via $P 2_{1} / m$. The Raman spectra associated with $P 2_{1} / m, P 2_{1}$ and $\mathrm{Cmcm}$ are too similar to identify the difference among those phases, reflecting closely related structures among these polymorphs [4-6,18,19,21]. A phase transition of $P 2_{1} \rightarrow$ $\mathrm{Cmcm}$ is a displacive-type phase transition accompanied only by slight displacements of $\mathrm{Sr} / \mathrm{Ba}$ and orientations of the $\mathrm{TaO}_{6}$ octahedra $[6,18,19,21]$, which causes a continuous variation in the unit-cell volumes across the phase transition. Therefore, the phase transition seems to be of the 2 nd order or of the 1st order near the 2 nd order with the low energy barrier between the two phases. Moreover, there is observed to be present $P 2_{1}$, a lower symmetry phase than $\mathrm{Cmcm}$, and the single phase at room temperature even if the particle size would be small due to the polymerizable complex synthesis. Usually, a higher symmetry phase appears when the particle size is small. Thus, the particle size has little influence on the phase transition temperature and an existing phase at room temperature [22].

The decomposition of each spectrum into bands was performed assuming Lorentzian band shapes. Most of the bands showed a frequency decrease with an increase in temperature as shown in Fig. 5. Relation describing dependence of the frequency of an $i$ th Raman band, $\omega_{i}$, on temperature, $T$, was obtained by fitting the values of $\omega_{i}$ by the least-squares rule, as follows:

$\omega_{i}=\omega_{i 0}+\left(\partial \omega_{i} / \partial T\right)_{P}\left(T-T_{0}\right)$

Here, $\omega_{i 0}$ refers to the $\omega_{i}$ value extrapolated at $T_{0}=298 \mathrm{~K}$ and $0.1 \mathrm{MPa}$. Because good linearity of each Raman band frequency with temperature was obtained in a temperature region from 573 to $1773 \mathrm{~K}$, the frequency data in this region were used to fit. The results are presented in Fig. 5 and Table I.

We discuss the present results here in terms of the temperature-varied parameter at constant pressure associated with the Gruneisen parameter. In the following equations, $\gamma_{i T}$ is the Gruneisen parameter of a considered mode $(i)$, related to the pressure variation at constant temperature, and $\gamma_{i P}$ the constant-pressure parameter with the temperature variation, defined in a way similar to $\gamma_{i T}[23,24]$.

$$
\begin{aligned}
& \gamma_{i T}=\left(\frac{\partial \ln \omega_{i}}{\partial \ln \rho}\right)_{T}=\frac{K_{T}}{\omega_{i}}\left(\frac{\partial \omega_{i}}{\partial P}\right)_{T} \\
& \gamma_{i P}=\left(\frac{\partial \ln \omega_{i}}{\partial \ln \rho}\right)_{P}=-\frac{1}{\alpha \omega_{i}}\left(\frac{\partial \omega_{i}}{\partial T}\right)_{P}
\end{aligned}
$$

Here, $\rho$ is the molar density, $\alpha$ the volume thermal expansion of a crystal, and $K_{T}$ the bulk modulus, which characterizes the incompressibility of structure. These parameters are crucial to assigning Raman bands. Values of $\gamma_{i P}$ for the Raman-active modes, estimated from Eq. (3), are summarized in Table I and Fig. 6. The values for $\omega_{i 0}$ and $\left(\partial \omega_{i} / \partial T\right)_{P}$ are estimated from the spectra in the temperature region from 573 to $1773 \mathrm{~K}$ in this work. The value of $\alpha \equiv 1 / V_{0}(\partial V / \partial T)_{P}$ used is $1.83 \times 10^{-5}$ $\mathrm{K}^{-1}$, which is reported for $\mathrm{Sr}_{2} \mathrm{Ta}_{2} \mathrm{O}_{7}$ in Ref. [25]. 
Table I Slopes of regression lines for frequencies as a function of temperature, and $\gamma_{i p}$ values for Raman-active modes.

\begin{tabular}{|c|c|c|c|}
\hline$i$ & $\omega_{i 0}\left(\mathrm{~cm}^{-1}\right)^{\dagger}$ & $\left(\frac{\partial \omega_{i}}{\partial T}\right)_{P}\left(\mathrm{~cm}^{-1} / \mathrm{K}\right)$ & $\gamma_{i P}(-)$ \\
\hline 1 & $60.4(2)$ & $-0.00495(16)$ & $4.48(16)$ \\
\hline 2 & $87(2)$ & $-0.006(2)$ & $4.0(14)$ \\
\hline 3 & $91.3(18)$ & $-0.0058(16)$ & $3.5(10)$ \\
\hline 4 & $126.1(5)$ & $-0.0105(3)$ & $4.55(17)$ \\
\hline 5 & $138.1(7)$ & $-0.0101(5)$ & $4.0(2)$ \\
\hline 6 & $201.2(10)$ & $-0.0139(7)$ & $3.8(2)$ \\
\hline 7 & $244.5(3)$ & $-0.0052(3)$ & $1.16(7)$ \\
\hline 8 & $286(2)$ & $-0.008(3)$ & $1.6(5)$ \\
\hline 9 & $298(3)$ & $-0.004(3)$ & $0.7(6)$ \\
\hline 10 & $327.6(14)$ & $-0.0096(14)$ & $1.6(2)$ \\
\hline 11 & $354(5)$ & $-0.016(4)$ & $2.4(6)$ \\
\hline 12 & $360(6)$ & $-0.006(5)$ & $0.8(8)$ \\
\hline 13 & $432(3)$ & $-0.008(3)$ & $1.0(4)$ \\
\hline 14 & $447(3)$ & $-0.011(3)$ & $1.4(3)$ \\
\hline 15 & $465(3)$ & $-0.008(2)$ & $0.9(3)$ \\
\hline 16 & $571(5)$ & $-0.012(5)$ & $1.1(5)$ \\
\hline 17 & $629.0(12)$ & $-0.0167(10)$ & $1.45(9)$ \\
\hline 18 & 793(13) & $-0.003(13)$ & $0.2(9)$ \\
\hline 19 & $843.14(15)$ & $-0.01591(11)$ & $1.031(7)$ \\
\hline 20 & $880(11)$ & $-0.009(11)$ & $0.5(7)$ \\
\hline
\end{tabular}

Raman and IR spectra of a crystalline solid generally can be described in terms of internal modes and external lattice modes. Modes derived from motions in the $\mathrm{TaO}_{6}$ group are considered to be the internal modes, to distinguish them from the external modes involving $\mathrm{Sr} / \mathrm{Ba}$ and Ta lattice vibrations. For the isolated $\mathrm{TaO}_{6}{ }^{7-}$ octahedral ion with the $O_{h}$ symmetry, the six normal vibrational modes are predicted to occur: two pure bond stretching vibrations of symmetry $A_{1 \mathrm{~g}}\left(\mathrm{v}_{1}\right)$ and $E_{\mathrm{g}}\left(\mathrm{v}_{2}\right)$, two interbond angle bending vibrations $v_{5}$ and $v_{6}$ of symmetry $F_{2 \mathrm{~g}}$ and $F_{2 \mathrm{u}}$, respectively, and finally, two remaining vibrations $v_{3}$ and $v_{4}$, considered as combinations of stretching and bending, both of $F_{1 \mathrm{u}}$ symmetry [26]. With a decrease in the symmetry of the $\mathrm{TaO}_{6}$ group in the crystalline lattice, all selection rules of the isolated $\mathrm{TaO}_{6}{ }^{7-}$ octahedron are broken. Thus, all these vibrations can become active with various intensities. However, the $\mathrm{TaO}_{6}$ internal modes of $\left(\mathrm{Sr}_{(1-0.15)} \mathrm{Ba}_{0.15}\right)_{2} \mathrm{Ta}_{2} \mathrm{O}_{7}$ originate from the $v_{1}, v_{2}, v_{3}, v_{4}, v_{5}$ and $v_{6}$ modes of the isolated ion. The $\gamma_{i P}$ parameter is proportional to the local thermal expansion coefficient of a considered bond $(i), \alpha_{i} ; \gamma_{i P} \propto \alpha_{i} / \alpha \quad$ [24]. For the higher frequency bands than $220 \mathrm{~cm}^{-1}$ ( $i=7$ to 20), the values of the $\gamma_{i P}$ parameter are smaller than for the lower frequency modes ( $i=1$ to 6$)$, which represents smaller $\alpha_{i}$ values, reflecting their relative incompressibility, weak expansivity, and strong bonds. The bulk modulus of the $\mathrm{TaO}_{6}$ octahedral units is generally larger than that of the crystal due to the covalent $\mathrm{Ta}-\mathrm{O}$ bonds. The thermal expansion coefficient of the $\mathrm{TaO}_{6}$ octahedral units is generally smaller than that of the crystal due to the same reason. These results and facts show that the higher frequency modes involve stretching and bending motions of the $\mathrm{Ta}-\mathrm{O}$ bonds in the $\mathrm{TaO}_{6}$ octahedra

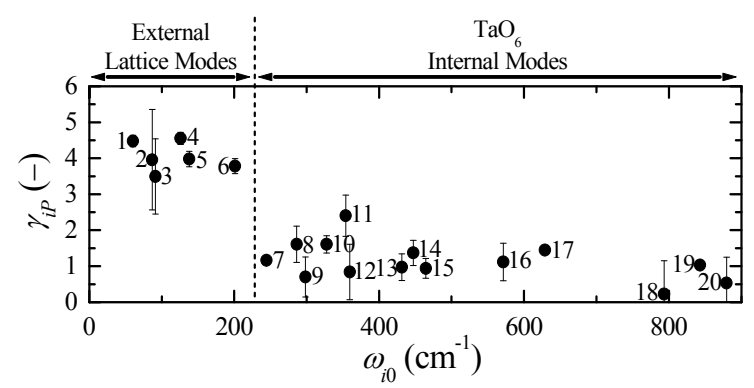

Fig. 6 Values of $\gamma_{i P}$ for considered Raman bands of $\left(\mathrm{Sr}_{0.85} \mathrm{Ba}_{0.15}\right)_{2} \mathrm{Ta}_{2} \mathrm{O}_{7}, i=1$ to 20 , in order of increasing frequency. The $\gamma_{i P}$ parameter is proportional to the local thermal expansion coefficient of a considered bond (i), $\alpha_{i}$; $\gamma_{i P} \propto \alpha_{i} / \alpha$.

(internal modes). On the other hand, $\gamma_{i P}$ is larger for the lower frequency bands of $i=1$ to 6 than for the bands of $i=7$ to 20 , which indicates that the oscillators have larger $\alpha_{i}$ values for the lower frequency bands, reflecting their relative compressibility, strong expansivity and weak bonds. The weaker bonds are caused by lower charged atoms and higher coordination numbers. Thus, these bands can be assigned to lattice vibrational modes (translational lattice modes of $\mathrm{Sr} / \mathrm{Ba}$ or $\mathrm{Ta}$ and/or rotational lattice modes of $\mathrm{TaO}_{6}$, etc.). Overall pressureand temperature-induced volume changes are greatly influenced by weaker bonds in the crystal structure. These assignments are supported by the deducible result from a calculation on the basis of a shell model [20].

For $\mathrm{Sr}_{2} \mathrm{Nb}_{2} \mathrm{O}_{7}$, most of the $\gamma_{i P}$ parameters of the internal modes are as large as those of the lattice modes, indicating that the chemical-bond strength of $\mathrm{Nb}-\mathrm{O}$ bonds is as weak as that of the external bonds [12]. The weak $\mathrm{Nb}-\mathrm{O}$ chemical bonds induce the larger distortions of the $\mathrm{NbO}_{6}$ octahedra and the static displacements of the niobium ions from the respective gravity centers of the $\mathrm{NbO}_{6}$ octahedra, which are as crucial as the static $\mathrm{NbO}_{6}$ octahedral rotations to decide deviation of the $\mathrm{Nb}-\mathrm{O}-\mathrm{Nb}$ bond angles from $180^{\circ}$. The deviation leads to a decrease in electron migration, which would decrease the photocatalytic performance. On the other hand, for Ba-substituted $\mathrm{Sr}_{2} \mathrm{Ta}_{2} \mathrm{O}_{7}$, all of the $\gamma_{i P}$ parameters of the internal modes are smaller than that of the lattice modes, reflecting stronger $\mathrm{Ta}-\mathrm{O}$ bonds than the lattice bonds. The substitution of $\mathrm{Ba}$ for $\mathrm{Sr}$ in $\mathrm{Sr}_{2} \mathrm{Ta}_{2} \mathrm{O}_{7}$ can induce the dipole moments in the $\mathrm{TaO}_{6}$ octahedra and the ferroelectric polarization in the whole crystal lattice without large deviation of the $\mathrm{Ta}-\mathrm{O}-\mathrm{Ta}$ bond angles from $180^{\circ}$ owing to the strong $\mathrm{Ta}-\mathrm{O}$ bonds. The dipole moments and the ferroelectric polarization act for the suppression of recombination between electrons and holes, and then enhance the photocatalytic performance.

\section{CONCLUSIONS}

In the present study, Raman spectra of $15 \mathrm{~mol} \%$ Ba-substituted strontium tantalate were successfully obtained at temperatures up to $1773 \mathrm{~K}$ for the first time, using a system of UV Raman spectroscopy. At room temperature, the SHG signal was clearly observed in the 
sample of $\left(\mathrm{Sr}_{1-x} \mathrm{Ba}_{x}\right)_{2} \mathrm{Ta}_{2} \mathrm{O}_{7}: \quad x=0.15$, indicating non-centrosymmetry due to displacement of cations and rotation of oxygen octahedra from an ideal layered perovskite structure with $\mathrm{Cmcm}$. Thus, the space group of the sample of $x=0.15$ is suggested to be $P 2_{1}$. The Raman spectra did not change significantly with an increase in temperature, despite the $P 2_{1} \rightarrow \mathrm{Cmcm}$ transition. The Raman spectra associated with the $P 2_{1} / m$, $P 2_{1}$, and $\mathrm{Cmcm}$ phases were very similar, reflecting closely related structures among these polymorphs.

The present results were discussed in terms of the constant-pressure parameter with temperature variation, $\gamma_{i P}$, defined in a similar way to the Gruneisen parameter. For the Raman bands located above $220 \mathrm{~cm}^{-1}$, the values of the parameter $\gamma_{i p}$ are smaller than for the lower frequency modes, indicating that these higher frequency modes are assigned to the internal vibrations of the $\mathrm{TaO}_{6}$ octahedra. The smaller values of $\gamma_{i P}$ for the $\mathrm{TaO}_{6}$ internal modes reflect stronger chemical-bond strength of $\mathrm{Ta}-\mathrm{O}$ bonds than the external modes involving $\mathrm{Sr}$ and $\mathrm{Ta}$ lattice vibrations. The strong $\mathrm{Ta}-\mathrm{O}$ chemical bonds cause the smaller distortions of the $\mathrm{TaO}_{6}$ octahedra and the smaller static displacements of the tantalum ions from the respective gravity centers of the $\mathrm{TaO}_{6}$ octahedra. Due to the strong $\mathrm{Ta}-\mathrm{O}$ chemical bonds, the dipole moments in the $\mathrm{TaO}_{6}$ octahedra and the ferroelectric polarization in the whole crystal lattice can be induced by the $\mathrm{Ba}$ substitution without large deviation of the $\mathrm{Ta}-\mathrm{O}-\mathrm{Ta}$ bond angles from $180^{\circ}$. Introducing of these electric fields would increase the photocatalytic performance by the suppression of recombination between electrons and holes. Ultraviolet Raman technique would open a new door for powerful tools that can be used to estimate chemical bonds at high temperature where accurate thermochemical measurement cannot be achieved. This study would be useful to clarify the mechanism of photocatalytic reaction for the improvement of the performance.

\section{ACKNOWLEDGMENT}

The authors thank Professor Kazuo Kasatani at Yamaguchi University for his help on the SHG measurement. Thanks are also due to Mr. Kensuke Morita at Yamaguchi University for his sample preparation. The present work has been supported partly with Collaborative Research Project of Materials and Structures Laboratory at Tokyo Institute of Technology.

\section{REFERENCES}

[1] A. Kudo, H. Kato, and S. Nakagawa, J. Phys. Chem. $B, 104$ [3], 571-75 (2000)

[2] M. Yoshino, M. Kakihana, W. S. Cho, H. Kato, and A. Kudo, Chem. Mater., 14 [8], 3369-76 (2002)

[3] A. Kozu, H. Fujimori, K. Kim, K. Oshiro, S. Yamamoto, Y. Sakata, and H. Imamura, Mater. Sci. Forum, 534-536, 1481-84 (2007)

[4] N. Yamamoto, M. Nakamura, K. Yagi, and K. Ohi, J. Phys. Soc. Jpn., 49, Suppl. B, 95-97 (1980)

[5] N. Yamamoto, K. Yagi, G. Honjyo, M. Kimura, and T. Kawamura, J. Phys. Soc. Jpn., 48 [1], 185-91 (1980)

[6] N. Ishizawa, F. Marumo, T. Kawamura, and M. Kimura, Acta Cryst., B32 [9], 2564-66 (1976)

[7] S. Kojima, K. Ohi, and T. Nakamura, Solid State
Commun., 35 [1], 79-81 (1980)

[8] Y. Akishige and K. Ohi, J. Phys. Soc. Jpn., 61 [4], 1351-56 (1992)

[9] M. Yashima, M. Kakihana, R. Shimidzu, H. Fujimori, and M. Yoshimura, Appl. Spectrosc., 51 [8], 1224-28 (1997)

[10] H. Fujimori, M. Kakihana, K. Ioku, S. Goto, and M. Yoshimura, Appl. Phys. Lett., 79 [7], 937-39 (2001)

[11] H. Fujimori, H. Komatsu, K. Ioku, S. Goto, and M. Yoshimura, Phys. Rev. B, 66 [6], 064306 (5 pages) (2002)

[12] I. Kawanishi, H. Fujimori, and N. Matsushita, Trans. Mat. Res. Soc. Jpn., 42 [6], 145-49 (2017)

[13] M. Kakihana, J. Sol-Gel Sci. Technol., 6 [1], 7-55 (1996)

[14] S. K. Kurtz and T. T. Perry, J. Appl. Phys., 39 [8], 3798-813 (1968)

[15] H. Fujimori, M. Yashima, M. Kakihana, and M. Yoshimura, Phys. Rev. B, 61 [6], 3971-74 (2000)

[16] H. Fujimori, H. Toya, K. Ioku, S. Goto, and M. Yoshimura, Chem. Phys. Lett., 325 [4], 383-88 (2000)

[17] H. Fujimori, M. Yashima, M. Kakihana, and M. Yoshimura, J. Am. Ceram. Soc., 84 [3], 663-65 (2001)

[18] M. Gasperin, Acta Cryst., B31 [8], 2129-30 (1975)

[19] N. Ishizawa, F. Marumo, S. Iwai, M. Kimura, and T. Kawamura, Acta Cryst., B36 [4], $763-66$ (1980)

[20] K. Ito and K. Ohi, J. Kor. Phys. Soc., 32, S545-S547 (1998)

[21] I. Kawanishi, H. Fujimori, and M. Yashima, to be submitted.

[22] H. Fujimori, D. Yahata, N. Yamaguchi, D. Ikeda, K. Ioku, and S. Goto, J. Ceram. Soc. Japan, 109 [5], 391-95 (2001)

[23] P. S. Peercy and B. Morosin, Phys. Rev. B, 7 [6], 2779-86 (1973)

[24] P. Gillet, F. Guyot, and J.-M. Malezieux, Phys. Earth Planet. In., 58 [2-3], 141-54 (1989)

[25] V. V. Kochetkov, N. A. Zakharov, S. Yu. Stefanovich, and Yu. N. Venevtsev, Sov. Phys. Crystallogr., 24 [5], 610-13 (1979)

[26] G. Herzberg, "Infrared and Raman Spectra of Polyatomic Molecules", Van Nostrand Reinhold, New York (1945) pp. 122-23

(Received November 21, 2017; Accepted December 23, 2017; Published Online February 1, 2018) 